\title{
Modelling Luminous-Blue-Variable Isolation
}

\author{
Mojgan Aghakhanloo, ${ }^{1 \star}$ Jeremiah W. Murphy, ${ }^{1} \dagger$ Nathan Smith,${ }^{2}$ \\ and Renée Hložek ${ }^{3}$ \\ ${ }^{1}$ Physics, Florida State University, 77 Chieftan Way, Tallahassee, FL 32306, USA \\ ${ }^{2}$ Steward Observatory, 933 N.Cherry Ave, Tucson, AZ 85719, USA \\ ${ }^{3}$ Dunlap Institute for Astronomy and Astrophysics, University of Toronto, 50 St. George Street, Toronto, Ontario, Canada M5S 3H4
}

28 September 2017

\begin{abstract}
Observations show that luminous blue variables (LBVs) are far more dispersed than massive O-type stars, and Smith \& Tombleson suggested that these large separations are inconsistent with a single-star evolution model of LBVs. Instead, they suggested that the large distances are most consistent with binary evolution scenarios. To test these suggestions, we modelled young stellar clusters and their passive dissolution, and we find that, indeed, the standard single-star evolution model is mostly inconsistent with the observed LBV environments. If LBVs are single stars, then the lifetimes inferred from their luminosity and mass are far too short to be consistent with their extreme isolation. This implies that there is either an inconsistency in the luminosityto-mass mapping or the mass-to-age mapping. In this paper, we explore binary solutions that modify the mass-to-age mapping and are consistent with the isolation of LBVs. For the binary scenarios, our crude models suggest that LBVs are rejuvenated stars. They are either the result of mergers or they are mass gainers and received a kick when the primary star exploded. In the merger scenario, if the primary is about $19 M_{\odot}$, then the binary has enough time to wander far afield, merge and form a rejuvenated star. In the mass-gainer and kick scenario, we find that LBV isolation is consistent with a wide range of kick velocities, anywhere from 0 to $\sim 105 \mathrm{~km} / \mathrm{s}$. In either scenario, binarity seems to play a major role in the isolation of LBVs.
\end{abstract}

Key words: binaries: general -stars: evolution -stars: massive-stars: variables: general

\section{INTRODUCTION}

Stellar mass is one of the primary characteristics that determine a star's evolution and fate (Woosley \& Heger 2015); therefore, understanding mass-loss is important in developing a complete theory of stellar evolution. Yet, understanding the physics and relative importance of steady and eruptive mass-loss in the most massive stars remains a major challenge in stellar evolution theory. There has been substantial progress in understanding mass-loss via steady linedriven winds of hot stars (Kudritzki \& Puls 2000; Puls et al. 2008), and this effect is included in stellar evolution models (Vink et al. 2001; Woosley et al. 2002; Meynet \& Maeder 2005; Martins \& Palacios 2013). However, the mass-loss rates of red supergiants (RSGs) and the role of eruptive mass-loss remain unclear, and the influence on stellar evolution remains uncertain (Smith \& Owocki 2006; Smith 2014).

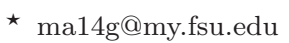

† jwmurphy@fsu.edu
The luminous blue variable (LBV) is one such poorly constrained class of eruptive stars.

LBVs are luminous, unstable massive stars that suffer irregular variability and major mass-loss eruptions (Humphreys \& Davidson 1994). The mechanism of these eruptions and the demographics of which stars experience these is poorly constrained (Smith et al. 2011; Smith 2014). The traditional view has been that most stars above 25$30 M_{\odot}$ pass through an LBV phase in transition from core $\mathrm{H}$ burning to He burning. In this brief phase, they experience eruptive mass-loss as a means to transition from a hydrogen-rich star to an H-poor Wolf-Rayet (WR) star (Humphreys \& Davidson 1994). In this scenario, LBVs experience high mass-loss due to an unknown instability, which may be driven by a high luminosity-to-mass (L/M) ratio, near the Eddington limit (Humphreys \& Davidson 1994; Vink 2012). However, a high L/M ratio may not be sufficient to explain LBV eruptions. Instead, the instability may require rare circumstances such as binary interactions (Smith \& Tombleson 2015).

Smith \& Tombleson (2015) noted that LBVs are iso- 
lated, and they proposed that binary interaction is important in LBV evolution and gives rise to their isolation. If LBVs mark a brief transitional phase at the end of the main sequence and before core-He burning WR stars, then they should be found near other massive O-type stars. However, Smith \& Tombleson (2015) found that LBVs are quite isolated from O-type stars, and even farther away from $\mathrm{O}$ stars than the WR stars are. Given their isolation, Smith \& Tombleson (2015) concluded that the LBV phenomenon is inconsistent with a single-star scenario and is most consistent with binary scenarios. In some respects, there was already earlier evidence that the simple LBV-toWR-to-SN mapping is not entirely accurate (Smith et al. 2007, 2008). For example, Kotak \& Vink (2006) proposed an LBV and supernova (SN) connection. Kotak \& Vink (2006) suggested that modulations in the radio light curve of $\mathrm{SNe}$ $2003 \mathrm{bg}$ and $1998 \mathrm{bw}$ reflected variations in the mass-loss rate similar to S Dor variations. In other cases, some Type IIn SNe may have LBV-like progenitors based on pre-SN massloss properties (mass, speed, $\mathrm{H}$ composition). For example, Ofek et al. (2013) reported a pre-supernova outburst $40 \mathrm{~d}$ before the Type IIn supernova SN 2010mc. Even though the progenitor of SN 2010mc was not directly identified as an LBV such an outburst is consistent with rare giant eruptions of LBVs. However, there has never been a direct connection between LBVs and Type IIn SNe. Instead, the connection is circumstantial in that narrow lines of Type IIn imply significant mass-loss from the progenitor, and even when the progenitor has been observed to vary, there are generally not enough observations to definitively classify a progenitor as an LBV. On the other hand, the isolation of directly identified LBVs provides a stronger constraint on their evolution (Smith \& Tombleson 2015).

In this paper, we constrain whether single-star or binary models are required to explain LBV isolation. We do this by developing simple models for the dispersal of massive stars on the sky. Our model is general and we designed it to have very few parameters. This simplicity and generalizability enable us to constrain the spatial and dynamic distributions of many stellar types. In this paper, we focus this generalized approach to model spatial distributions of early, mid and late O-type stars and most importantly LBVs. In particular, we use our models to constrain whether LBV isolation is consistent with single-star evolution or binary evolution.

Part of the reason that LBVs are poorly understood is that there are few examples. There are only 10 unobscured in our Galaxy and 19 known in the nearest galaxies, the Large Magellanic Cloud (LMC) and Small Magellanic Cloud (SMC; Smith \& Tombleson 2015). Even this small sample includes 'candidate' LBVs (see below). Classifying various stars as LBVs or candidates can be somewhat controversial (Humphreys \& Davidson 1994; Weis 2003; Vink 2012); here we summarize their basic characteristics. LBVs are luminous, blue massive stars with irregular or eruptive photometric variability. Stars that resemble LBVs in their physical properties and spectra, but lack the tell-tale variability, are usually called 'LBV candidates'. The reason they are sometimes grouped together is that it is suspected that the LBV instability may be intermittent, so that candidates are temporarily dormant LBVs (Smith et al. 2011; Smith 2014). The LBV candidates in Smith \& Tombleson (2015) have shell nebulae that are thought to be indicative of past eruptive mass-loss.

Although the signature eruptive variability of LBVs was identified long ago, the physical theory of LBV eruptions is not yet clear. For the most part, LBVs seem to experience two classes of eruptions: S Doradus (or S Dor) eruptions (1-2 mag) and giant eruptions ( $\geq 2 \mathrm{mag})$.

$\mathrm{S}$ Doradus variables take their namesake from the prototypical LBV S Doradus (van Genderen 2001). During S Dor outbursts, LBVs make transitions in the HR diagram (HRD) from their normal, hot quiescent state to lower temperatures (going from blue to red). In its quiescent state, an LBV has the spectrum of a B-type supergiant or a late Oftype/WN star (Walborn 1977; Bohannan \& Walborn 1989). In this state, LBVs are fainter (at visual wavelengths) and blue with temperatures in the range of 12000-30000 K (Humphreys \& Davidson 1994). In their maximum visible state, their spectrum resembles an F-type supergiant with a relatively constant temperature of $\sim 8000 \mathrm{~K}$. S Dor events were originally proposed to occur at constant bolometric luminosity (Humphreys \& Davidson 1994). So a change in temperature implies a change in the photospheric radius, $L=4 \pi \sigma R^{2} T^{4}$. Humphreys \& Davidson (1994) suggested that the eruption is so optically thick that a pseudophotosphere forms in the wind or eruption. However, quantitative estimates of mass-loss rates show that they are too low to form a large enough pseudo-photosphere (de Koter et al. 1996; Groh et al. 2009). Similar studies also imply that the bolometric luminosity is not strictly constant (Groh et al. 2009). Instead, it has been suggested that the observed radius change of the photosphere can be a pulsation or envelope inflation driven by the Fe opacity bump (Gräfener et al. 2012).

The other distinguishing type of variability is in the form of giant eruptions like the 19th century eruption of $\eta$ Car (Smith et al. 2011). The basic difference from $\mathrm{S}$ Dor events is that giant eruptions show a strong increase in the bolometric luminosity and are major eruptive mass loss events, whereas S Dor eruptions occur at roughly constant luminosity and are not major mass-loss events. The mass-loss rate at S Dor maximum is of the order of $10^{-4} M_{\odot} y r^{-1}$ or less (Wolf 1989; Groh et al. 2009). On the other hand, giant eruption mass loss rate is of the order of $10^{-1}-1 M_{\odot} y r^{-1}$ (Owocki et al. 2004; Smith \& Owocki 2006; Smith 2014). It is unlikely that a normal linedriven stellar wind is responsible for the giant eruptions because the material is highly dense and optically thick (Owocki et al. 2004; Smith \& Owocki 2006). Instead, giant eruptions must be continuum-driven super-Eddington winds or hydrodynamic explosions (Smith \& Owocki 2006). Both of these lack an explanation of the underlying trigger; the super-Eddington wind relies upon an unexplained increase in the star's bolometric luminosity, whereas the explosive nature of giant eruptions would require significant energy deposition. There is much additional discussion about the nature of LBV giant eruptions in the literature (Humphreys \& Davidson 1994; Owocki et al. 2004; Smith \& Owocki 2006; Smith et al. 2011; Smith 2014).

Smith \& Tombleson (2015) highlighted a result that changes the emphasis on the most likely models. They found that compared to O stars, LBVs are isolated in the Milky Way and the Magellanic Clouds. Moreover, they found that 
LBVs appear to have a much larger separation than even WR stars, which are thought to be the descendants of LBVs. They concluded that the single-star model is inconsistent with the statistical properties of LBV isolation. At a minimum, they suggested that LBV isolation may require binary evolution for a large fraction of LBVs if not all.

Humphreys et al. (2016) put forth a different interpretation of LBV locations, suggesting that they do not rule out the single-star scenario. They noted that the sample in Smith \& Tombleson (2015) is a mixture of less luminous LBVs, more luminous classical LBVs and unconfirmed LBVs, and they proposed that separating them alleviates the conflict with single-star models. From their point of view, the single-star hypothesis still works because (1) the three most luminous stars of the sample that are classical LBVs (with initial masses greater than $50 M_{\odot}$ ) have a distribution similar to late O-type stars, and (2) the less luminous LBVs (with initial mass $\sim 25-40 M_{\odot}$ ) are not associated with any $\mathrm{O}$ stars, but have a distribution similar to RSGs, which could be consistent with them being single stars on a postRSG phase. They strongly suggested that one separates the LBVs into two categories by luminosity for future statistical tests. Moreover, Humphreys et al. (2016) criticized that five of the LMC stars (R81, R126, R84, Sk-69271 and R99) are neither LBVs nor candidates.

However, Smith (2016) showed that even using the LBV sample subdivided as Humphreys et al. (2016) preferred does not change the result that LBVs are too isolated for single-star evolution (overlooking the lack of statistical significance). The most massive LBVs appear to be associated on the sky with late O-type dwarfs (point 1 above), which, however, have initial masses less than half of the presumed initial masses of the classical LBVs. Similarly, the lower luminosity LBVs have a similar distribution to RSGs, but these RSGs are dominated by stars of 10-15 $M_{\odot}$ (Smith 2016).

Humphreys et al. (2016) also stated that the observed LBV velocities seem to be too small to be consistent with the kicked mass-gainer scenario, but Smith (2016) pointed out that without a quantitative model for the velocity distributions, it would be difficult to rule anything in or out. In this paper, we will show that both high- and low-luminosity LBVs and LBV candidates have larger separations than one would expect, and in Section 4.4 and Fig. 10 we show that a wide range of kick velocities are consistent with the large separations.

The main goal of this paper is to quantitatively constrain whether the relative isolation of LBVs is inconsistent with a single-star evolution model. We begin by reproducing and verifying Smith \& Tombleson (2015) results (Section 2). In Section 3, we introduce a simple model for young stellar clusters and their passive dissolution. To test this model, we also compare the separations between $\mathrm{O}$ stars for the model and observations; we find that the model reproduces some general properties of the spatial distribution of massive stars, but it is lacking in other ways. Since we constructed the simplest model possible, this implies that we may improve the dispersion model and learn even more about the evolution of massive stars. Then in Section 4, we present the primary consideration of this paper; we compare singlestar evolution and binary evolution in the context of cluster dissolution, and we find that the single-star evolution sce- nario is inconsistent for initial masses appropriate for LBV luminosities. We discuss two binary evolution channels that are consistent with the relative isolation of LBVs. We then summarize, and we discuss future observations to further constrain the binary models (Section 5).

\section{OBSERVATIONS}

In the following sections, we explore which theoretical models are most consistent with the data, but before that, we clearly define, analyse and characterize the data in this section. First, we reproduce and verify the results of Smith \& Tombleson (2015). Secondly, we further characterize the data, noting that the distributions of nearest neighbours are lognormal. Since lognormal distributions have very few parameters, this restricts the complexity and parameters of our models in Section 3.

Smith \& Tombleson (2015) found that LBVs are much more isolated than O-type or WR stars, suggesting that LBVs are not an intermediary stage between these two evolutionary stages. In particular, they found that on average, the distance from LBVs to the nearest $\mathrm{O}$ star is quite large $(0.05 \mathrm{deg})$. For comparison, the average distance from early $\mathrm{O}$ stars to the nearest $\mathrm{O}$ star is $0.002 \mathrm{deg}$, and from mid and late $\mathrm{O}$ stars are 0.008 and $0.010 \mathrm{deg}$ respectively. If single early- and mid-type $\mathrm{O}$ stars are indeed the main-sequence progenitors of LBVs, then one would expect the spatial separations between LBVs and other $\mathrm{O}$ stars to be not too different from the separation between early- and mid-type O stars. However, the LBV separations are an order of magnitude farther than the early- and mid-type separations. In fact, the LBV separations are five times larger than even the late-type $\mathrm{O}$ stars, which live longer and can in principle migrate farther.

Smith \& Tombleson (2015) quantified the difference in the distributions of separations by using the KolmogorovSmirnov (KS) test. Comparing the distributions of LBVs to early, mid and late types gives $P$-values of $5.5 \mathrm{e}-9,1.4 \mathrm{e}^{-}$ 4 and $4.4 \mathrm{e}-6$, respectively. These values imply that $\mathrm{O}$-stars and LBV distributions are quite different. If true, then these results have profound consequences for our understanding of LBVs and their place in massive star evolution. In fact, Smith \& Tombleson (2015) suggested that the most natural explanation is that LBVs are the result of extreme binary encounters. Later we will test this assertion, but for now we reproduce and verify their results.

To verify the results of Smith \& Tombleson (2015), we first define the data. The data consist of two main parts: LBVs and O stars. Their sample includes WR stars, sgB[e] stars and RSGs too, but we do not discuss them here because at the moment, we want to keep our models in Sections 3 and 4 simple and we will focus just on LBVs and O stars. Their LBV samples include 16 stars in the LMC, and three stars in the SMC. They did not consider Milky Way LBVs because the distances and intervening line-of-sight extinction in the plane of the Milky Way are uncertain (Smith \& Stassun 2017). In their study, they included LBV candidates with a massive CSM (circumstellar medium) shell that likely indicates a previous LBV-like giant eruption. LBVs and their important parameters are summarized in Table 1.

The masses of LBVs that are in Table 1 are uncertain; 
Table 1. List of LBVs and LBV candidates adapted from Smith \& Tombleson (2015). For the stars in the SMC, we rescale their angular separation by 1.2 as if they are located at the distance of the LMC. Parentheses in the name represent LBV candidates and parentheses in mass column specify the LBVs with relatively poorly constrained luminosity and mass.

\begin{tabular}{lccr}
\hline LBV (name) & Galaxy (name) & $S(\operatorname{deg})$ & $M_{\text {eff }}\left(M_{\odot}\right)$ \\
\hline R143 & LMC & 0.00519 & 60 \\
R127 & LMC & 0.00475 & 90 \\
S Dor & LMC & 0.0138 & 55 \\
R81 & LMC & 0.1236 & $(40)$ \\
R110 & LMC & 0.2805 & 30 \\
R71 & LMC & 0.4448 & 29 \\
MWC112 & LMC & 0.0892 & $(60)$ \\
R85 & LMC & 0.0252 & 28 \\
(R84) & LMC & 0.1575 & 30 \\
(R99) & LMC & 0.0412 & 30 \\
(R126) & LMC & 0.0358 & $(40)$ \\
(S61) & LMC & 0.1432 & 90 \\
(S119) & LMC & 0.3467 & 50 \\
(Sk-69142a) & LMC & 0.0522 & 60 \\
(Sk-69279) & LMC & 0.0685 & 52 \\
(Sk-69271) & LMC & 0.040 & 50 \\
HD5980 & SMC & 0.0191 & 150 \\
R40 & SMC & 0.1112 & 32 \\
(R4) & SMC & 0.0160 & $(30)$ \\
\hline
\end{tabular}

specifically, the uncertainty in masses due to distance uncertainties is at least $8 \%$, but the systematic uncertainties due to the stellar evolution modelling are likely much larger. Currently, it is difficult to adequately quantify these uncertainties. None of them are kinematic mass measurements. Rather, they are based upon inferring the mass by comparing their colour and magnitude in the HRD with evolutionary tracks of various masses. In this modelling, the two main sources of uncertainties are modelling the uncertain physics of late-stage evolution and distance. The distance uncertainty to the LMC is 3-4\% (Marconi \& Clementini 2005; Walker 2012; Klein et al. 2014), which would translate to a luminosity uncertainty of $6-8 \%$. However, the systematic uncertainties in modelling LBVs and their luminosities and colour are unknown and could easily be much larger than the distance uncertainty. Therefore, like Smith \& Tombleson (2015), we merely report rough estimates for the LBV masses in Table 1.

Smith \& Tombleson (2015) gathered the positions of Otype stars within $10^{\circ}$ projected radius of 30 Dor from SIMBAD data base. They also used the revised Galactic O-star Catalog (Maíz Apellániz et al. 2013) to check their O-star samples (not shown in their paper), but as they claimed this did not change their overall results. We collect the same O-star samples from SIMBAD data base.

After gathering the data, we find the distance from one star to the nearest O star. The bottom panel of Fig. 1 shows the resulting cumulative distributions (the top panel shows results of our modelling, which we discuss in Section 3); note that the distributions for LBV and O-type separations are quite distinct. For example, the $P$-value for the comparison of the LBV and the mid-type distributions is $2.8 \times 10^{-5}$. Our KS-test $P$-values are listed in Table 2 . We consider three KS tests. In one, we compare the separation for both confirmed
Table 2. The $P$-values for KS tests for the distributions of separation. We are comparing the separations between LBVs and $\mathrm{O}$ stars and the separations between $\mathrm{O}$ stars of various types. Broadly, we reproduce the results of Smith \& Tombleson (2015) who found that the distribution of separations between LBVs and the nearest $\mathrm{O}$ star is quite different from the distributions for the separations between $\mathrm{O}$ stars and the nearest $\mathrm{O}$ star. The second row shows the results of our KS tests between the LBV separations and the early-, mid- and late-type $\mathrm{O}$ stars. Our results are similar to those of (Smith \& Tombleson 2015, first row), first row. Like Smith \& Tombleson (2015) we obtain the positions of O stars and their rayet spectral types from SIMBAD. Smith \& Tombleson (2015) updated the spectral types with the Galactic O-star Catalog (Maíz Apellániz et al. 2013); however, we did not. This slight difference in spectral typing is what causes the modest difference in $P$-values. In either case, the LBV separations are inconsistent with any O-type separations. If we exclude the LBV candidates (third row), the conclusions remain the same, but the significance is greatly reduced.

\begin{tabular}{lccr}
\hline Data set & Early O & Mid O & Late O \\
\hline LBV+LBVc (Smith \& Tombleson 2015) & $5.5 \mathrm{e}-9$ & $1.4 \mathrm{e}-4$ & $6.4 \mathrm{e}-06$ \\
LBV+LBVc (this work) & $8.2 \mathrm{e}-08$ & $2.8 \mathrm{e}-05$ & $8.4 \mathrm{e}-05$ \\
LBV (this work) & $9.2 \mathrm{e}-04$ & $2.3 \mathrm{e}-02$ & $5.7 \mathrm{e}-02$ \\
LBVc (this work) & $6.4 \mathrm{e}-06$ & $5.1 \mathrm{e}-05$ & $2 \mathrm{e}-04$ \\
\hline
\end{tabular}

and candidate LBVs with O-star distribution. In the second, the LBV distribution only includes confirmed LBVs, and in the third, the LBV distribution only contains the candidates. When we include both confirmed and candidate LBVs, the LBV and O-star distributions are clearly not drawn from the same parent distribution. However, omitting LBV candidates reduces the distinctions between the distributions. One might argue that since LBVs represent a later evolutionary stage, then the spatial separations should be larger, and therefore, the distributions of early-type $\mathrm{O}$ stars and LBVs should not represent the same distribution. However, we will show in section 4 that the lifetimes of massive stars are far too short to explain these large discrepancies. In our initial assessment, we agree with Smith \& Tombleson (2015); the large separations present a challenge to the single-star evolution scenario. In the next sections, we will present theoretical models to quantify this inconsistency.

Before we constrain the models, note that the separation distributions are lognormal (see Fig. 2). In fact, this simple observation greatly restricts the complexity of the models that we may explore in the next sections. If a variable such as the separation between stars shows a lognormal distribution, then there are only two free parameters that describe the distribution, the mean and the variance. In addition, if the separation depends upon other variables such as a velocity distribution, then thanks to the central limit theorem, the separation distribution will only depend upon the mean of the variance of the secondary variables such as the velocity distribution. This means that we cannot propose overly complex models for the velocity distribution. We would only be able to infer the mean and the variance anyway. Fortunately, we may measure the separation for different types of $\mathrm{O}$ stars and other evolutionary stages. This means that we may infer the temporal evolution in addition to the mean and variance. Whatever models we propose, they cannot be too elaborate; we will only be able to infer the mean and variance of one quantity as a function of time. 


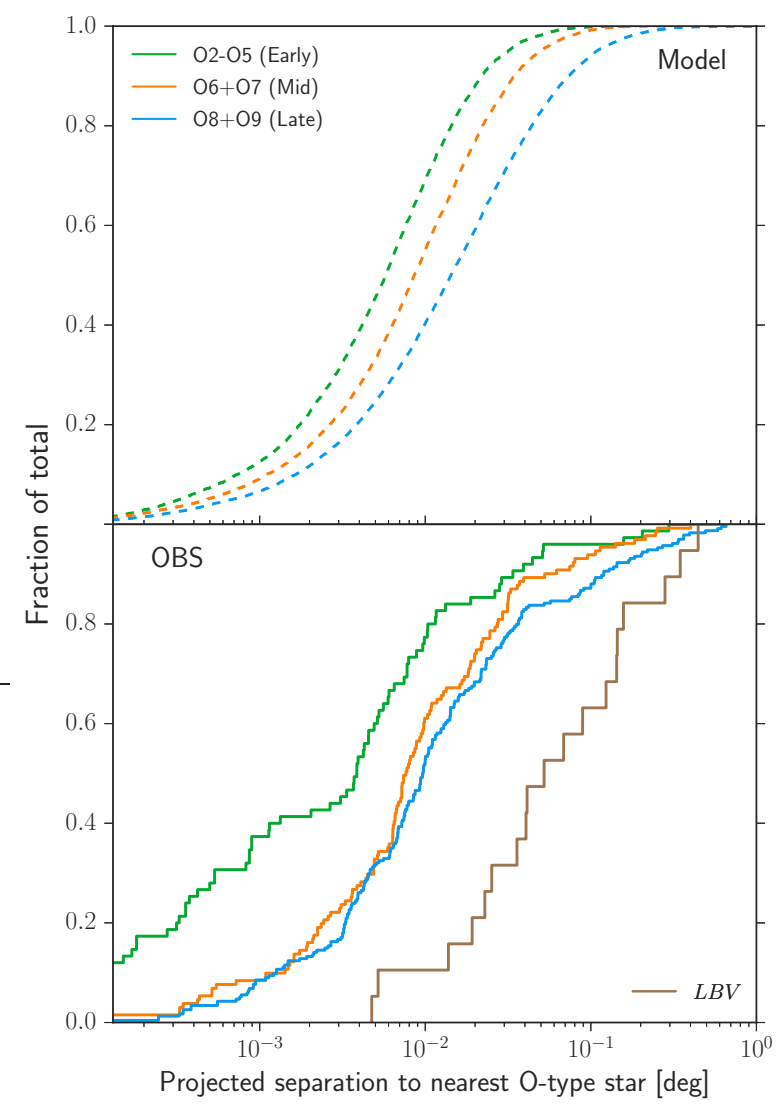

Figure 1. Cumulative distributions for the projected separation to the nearest $\mathrm{O}$ star. The top panel represents the modelled distribution for $\mathrm{O}$ stars and the bottom panel represents the data for both O stars and LBVs. Later, we will use the modelled O-star distributions to devise a general dispersion model, which we use to model the LBV separations (see Section 4). Broadly, the model reproduces the observations; both show a lognormal distribution, and the average separation increases with spectral-type because the later spectral type last longer.

\section{A GENERIC MODEL FOR THE SPATIAL DISTRIBUTION OF THE STARS IN A PASSIVE DISPERSAL CLUSTER}

In order to model the relative isolation of LBVs, we need to model the dissolution of clusters and associations of massive stars. For several reasons, we model the dissolution of young stellar clusters with a minimum set of parameters. For one, the O-star distributions are lognormal. Therefore, there are only a few parameters that describe the data that one may fit. The only data that we can reliably fit are the mean, variance and time evolution of the separations. So whatever models we develop, they should not be overly complex. Also, as far as we know, there are no simple self-consistent and tested models for the dissolution of clusters. Therefore, we propose a simple model of cluster dissolution and adapt it to consider two scenarios: cluster dissolution in the context of single-star evolution and cluster dissolution with close binary interactions. In this section, we present a cluster disper-

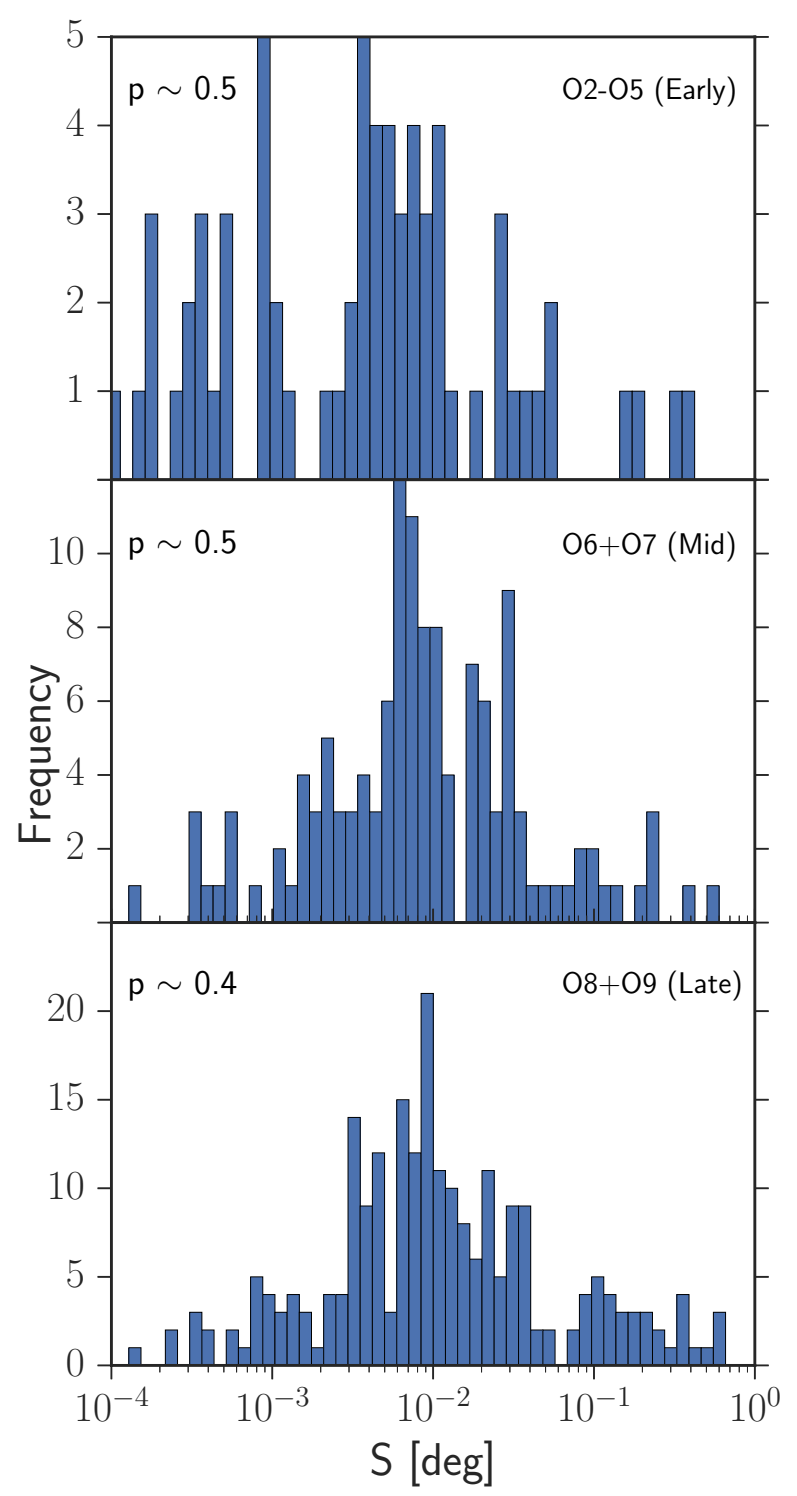

Figure 2. Normality test. The distributions of separations for early, mid, and late $\mathrm{O}$ stars are consistent with a lognormal distribution. In each plot, we show the probability, $p$, that the parent distribution is a lognormal distribution.

sal model considering only single-star evolution. While our dissolution models represent the spatial distributions reasonably well in certain respects, we note that our model fails to match the data in other ways. This implies that our model is missing something. In other words, we may be able to infer more physics about the dissolution of clusters from the simple spatial distribution of $\mathrm{O}$ stars. In the next section, we contrast the single-star model with a model that considers binarity.

Our main goal is to introduce a model for young stellar clusters that predicts the spatial distribution of massive stars, especially $\mathrm{O}$ stars. We start by considering the sim- 
plest model. In the following, we model the average distance to the nearest $\mathrm{O}$ star by nothing more than the passive dispersal of a cluster.

Before we dive into the details of the model, it is worth characterizing the scales of a typical cluster. We begin right after star formation ends and consider a system of gas and stars that is in virial equilibrium. In this case, we have $2 T+U=0$, where $T$ is the total thermal plus kinetic energy and $U$ is the gravitational potential energy. Initially, the system with total mass $M_{T}$ and radius $R$ is bound, and the stars have a velocity dispersion that scales as the gravitational potential of the entire system $\sigma_{v} \sim\left(G M_{T} / R\right)^{\frac{1}{2}}$. Then the system loses gas mass by some form of stellar feedback (UV radiation, stellar winds, etc.) and likely makes the stars unbound. If the system loses all of the gas quickly, then the stars will drift away with a speed roughly equal to the velocity dispersion when the cluster was bound. Hence, $v_{d} \sim \sigma_{v}$. All that is left to do is estimate $M_{T}$ and $R$. A typical cluster has $R \sim 4$ pc and about $40 \mathrm{O}$ stars; if only $\sim 1 \%$ of the gas in giant molecular clouds form stars (Krumholz \& Tan 2007), the total mass of the molecular cloud, $M_{T}$, is the order of $2 \times 10^{5} M_{\odot}$. Given these approximations, we estimate that the drift velocity is the order of $v_{d} \sim 13.5\left(\frac{M_{T}}{2 \times 10^{5} M_{\odot}} \frac{4 p c}{R}\right)^{\frac{1}{2}}$.

Next, we present a more specific dissolution model to convert this dispersal velocity into a distribution of separations as a function of time. Rather than using this estimate for the dispersal velocity, we will use the data and our model to infer the dispersal velocities. We propose a Monte Carlo model for the dissolution of the clusters. First, we randomly sample $N_{c l}$ clusters uniformly in time between 0 and $11 \mathrm{Myr}$. For each cluster, we draw a cluster mass from a distribution of cluster masses. Then, we estimate the total number of the stars $\left(N_{*}\right)$, and for each cluster, we draw a distribution of stellar masses $\left(M_{*}\right)$ from the Salpeter distribution.

First, we randomly select a total number of O stars ,$N_{*}$, for each cluster. The distribution from which we draw the size of each cluster is the Schechter function (Elmegreen \& Efremov 1997), $\frac{d N_{c l}}{d M_{c l}} \propto M_{c l}^{-2}$, where $M_{c l}$ is the mass of the cluster. However, we are most interested in the number of $\mathrm{O}$ stars for each cluster, so our first order of business is to express the Schechter function in terms of the number of $\mathrm{O}$ stars. The mass of the cluster is $M_{c l}=A \int_{M_{* 1}}^{M_{* 2}} M_{*}{ }^{-1.35}$, where $M_{* 1}$ and $M_{* 2}$ are the minimum and maximum masses of $\mathrm{O}$ star that we consider. In terms of this, the total number of $\mathrm{O}$ stars becomes

$N_{*}=\frac{M_{c l}}{\int_{M_{* 1}}^{M_{* 2}} M_{*}^{-1.35} d M_{*}} \int_{M_{* 1}}^{M_{* 2}} M_{*}^{-2.35} d M_{*}$.

Therefore, the total number of stars in the cluster is proportional to the mass of the cluster $\left(N_{*} \propto M_{c l}\right)$, and we can easily translate the distribution in mass to a distribution in the number of stars for each cluster, $\frac{d N_{c l}}{d N_{*}} \propto N_{*}^{-2}$. If $R_{*}$ is drawn from the uniform distribution between 0 and 1 , then the total number of stars in the cluster is

$N_{*}=\frac{1}{R_{*}\left(N_{* \max ^{-1}-N_{* \min }}{ }^{-1}\right)+N_{* \min }{ }^{-1}}$,

where $N_{* \max }$ and $N_{* \min }$ are the maximum and minimum number of the stars in the cluster.

For each star, we draw the mass from the Salpeter initial

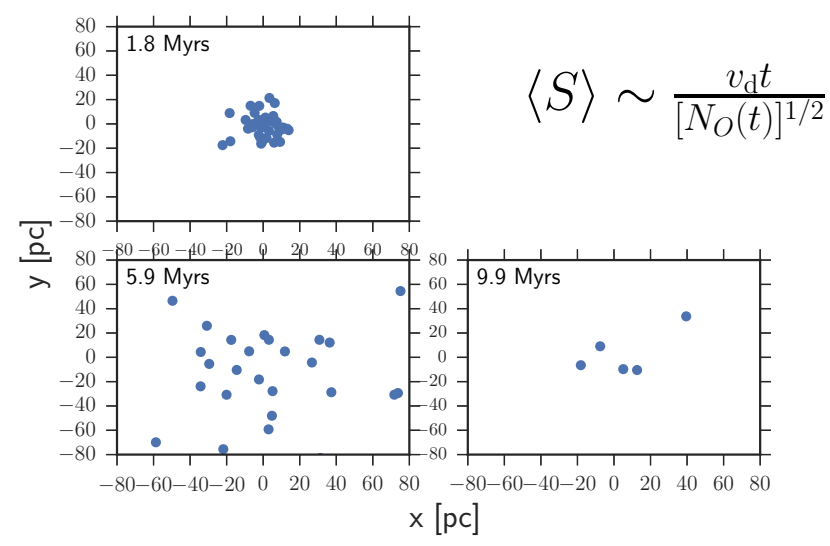

Figure 3. We propose a Monte Carlo model for the separations between $\mathrm{O}$ stars and LBVs by considering a random sample of dissolving clusters at random ages. Here we show the $\mathrm{O}$ stars of three randomly generated clusters, each with its own age. Note that the average separation between the $\mathrm{O}$ stars increases with age for two reasons. First, the separations increase as the cluster disperses with a drift velocity $v_{d}$ over time $t$. Secondly, O stars disappear as they evolve.

mass function (IMF),

$M_{*}=\left(\frac{1}{\left[R_{m}\left(M_{\max ^{-1.35}-M_{\min }}^{-1.35}\right)\right]+M_{\min }{ }^{-1.35}}\right)^{0.74}$,

where $R_{m}$ is a random number between 0 and 1 .

Having established the initial conditions, we now describe the evolution. The average separation between O stars depends upon how much the cluster has dispersed and how many $\mathrm{O}$ stars are left. So we need to model the dispersion of the $\mathrm{O}$ stars and their disappearance. Therefore, we need to model the spatial distribution (or spatial density) and time evolution of massive stars in a cluster. Once we establish the spatial distribution, we then calculate the separation between stars. The distribution of separations in essence is a convolution of the density function with itself. Because this is a multiplicative process, the central limit theorem implies a lognormal distribution. The central limit theorem also dictates that any underlying spatial distribution with a well-defined mean and variance results in a lognormal distribution. Therefore, we are free to choose a simple model for the spatial distribution, and we choose a Gaussian for the spatial distribution.

For the time evolution we assume that each cluster is passively dispersing with a typical velocity scale of $v_{d}$. Therefore, the characteristic size scale of the Gaussian spatial distribution is $\sigma=v_{d} t$. Given the assumption that stars are coasting then the individual velocities are $r / t$. With these assumptions, then the distribution of velocities is Gaussian too, $p(v)=\frac{t}{\sqrt{2 \pi \sigma^{2}}} e^{-r^{2} / 2 \sigma^{2}}$.

Another important aspect of modelling these clusters is to model the age and disappearance of massive stars. For the lifetimes, we use the results of single-star evolutionary models from the binary population synthesis code, BINARY_C (Izzard et al. 2004, 2006, 2009). Therefore, the average separation between stars goes up both because the cluster is dispersing and $\mathrm{O}$ stars are disappearing. Fig. 3 shows the spatial distribution of an example model at several ages. 
With our model defined, our first task is to constrain whether the average distances between LBVs and O stars are consistent with the passive dissolution of a cluster with single-star evolution. To compare our models to the data, we calculate the angular separations, assuming that the clusters are at the distance of the LMC. Furthermore, to be consistent with Smith \& Tombleson (2015), we subdivide the modelled O stars into early, mid and late types based upon their masses. To convert from mass to spectral type, we used Martins et al. (2005) data. Early-type O stars have masses greater than $34.17 M_{\odot}$, late-type $\mathrm{O}$ stars have masses $\leq 24.15$ $M_{\odot}$ and mid-type $\mathrm{O}$ stars have masses in between. In the next subsection, we test whether our passive single-star dissolution model is consistent with the data.

\subsection{COMPARING THE PASSIVE SINGLE-STAR DISSOLUTION MODEL WITH THE DATA}

Next, we compare the passive dissolution of single stars to the LMC and SMC nearest-neighbour distributions. Fig. 1 shows the cumulative distribution for the separations for our simple dissolution model (top panel) and for the observations (bottom panel). For illustration purposes, we set $v_{d}$ to $14.5 \mathrm{~km} / \mathrm{s}$, making the modelled distribution have about the same mean as the data. So far, our passive dissolution model is in good agreement with observations. Both the model and observations show a lognormal distribution in separations, and the average separation increases with spectral type, which is expected since later $\mathrm{O}$ stars live longer and have more time to disperse.

Because the distributions are lognormal, there are only two parameters that describe the distribution, the mean and std. deviation. Therefore, we investigate how our model reproduces these two distribution characteristics. The primary parameter in our model is $v_{d}$, so in Fig. 4 we plot the mean (bottom panel) and std. deviation (top panel) as a function of $v_{d}$. The dashed lines represent the modelled mean and std. deviation, and the solid bands indicate the observed values. The vertical axes in Fig. 4 are $\overline{\mu_{S}}$ and $\overline{\sigma_{S}}$. First, we calculate the mean and std. deviation in log; then, we calculate $\overline{\mu_{S}}=10^{\mu(\log S)}$ and $\overline{\sigma_{S}}=10^{\sigma(\log S)}$. The solid bands provide some estimate of uncertainty in our inferred drift velocity, we bootstrap the observations, giving a variance for both the mean and std. deviation.

We draw three main conclusions from Fig. 4. For one, the drift velocities that we infer by comparing our simple model with the data are roughly what we would expect; see our order-of-magnitude estimate in Section 3. Secondly, we infer larger drift velocities for the late-type $\mathrm{O}$ stars (10-12 $\mathrm{km} / \mathrm{s})$ in comparison to early-type stars $(6-8 \mathrm{~km} / \mathrm{s})$. However, this trend is not monotonic; the mid-type $\mathrm{O}$ stars have an inferred drift velocity $(14-16 \mathrm{~km} / \mathrm{s})$ that is similar to but slightly higher than the late-type O stars. Thirdly, our simple model is not able to reproduce the variance in the distributions. This implies that something is missing from our model. In other words, there is more that we can learn about the evolution of massive stars in clusters from their spatial distributions. Despite the shortcomings, the model is able to reproduce the average separations with reasonable drift velocities. Therefore, we proceed with our analyses under these caveats.
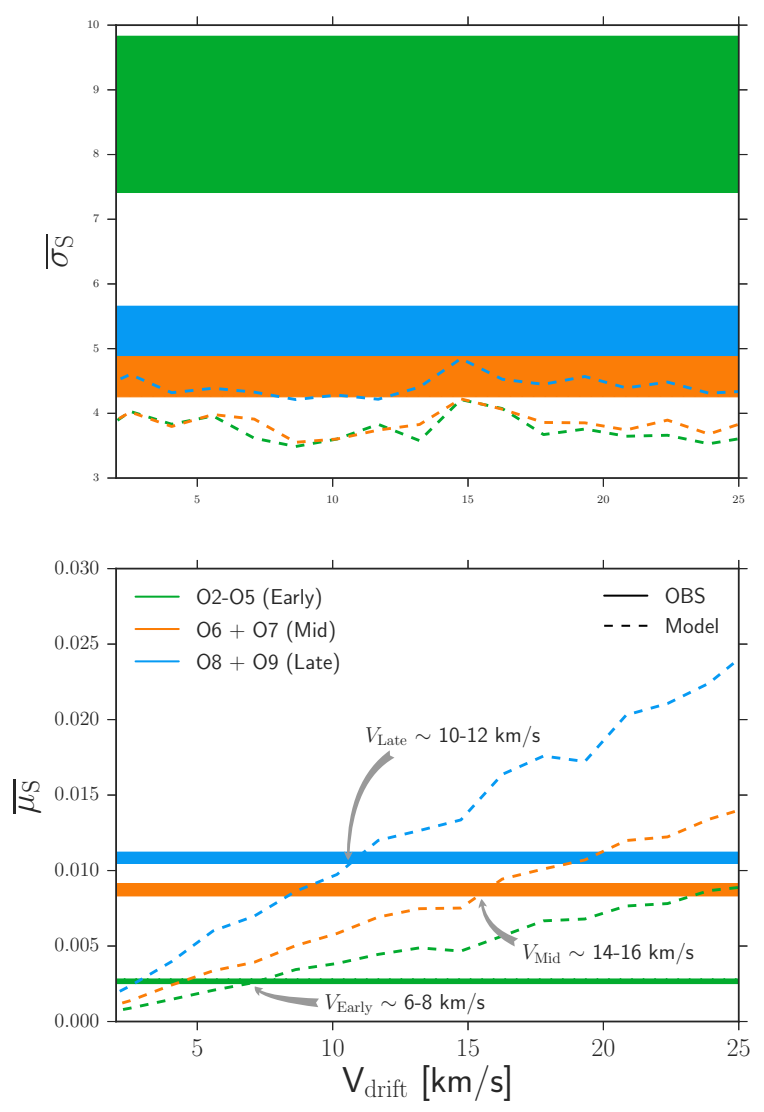

Figure 4. The mean (bottom panel) and std. deviation (top panel) distance to the nearest neighbour versus drift velocity. We calculate the mean and std. deviation in log first; then, we calculate the $\overline{\mu_{S}}=10^{\mu}(\log S)$ and $\overline{\sigma_{S}}=10^{\sigma}(\log S)$

. In both panels, dashed lines represent the passive dissolution model and solid lines represent the observational data (Smith \& Tombleson 2015). We highlight three main conclusions. (1) The drift velocities that we infer by comparing our simple model with the data are roughly what we estimated in Section 3. (2) We infer larger drift velocities for the later type O stars, implying that binary evolution and kicks may be important. (3) The passive dissolution model is not able to reproduce the variance in the distributions, which implies missing physics from our model. In other words, there is room to improve our model and learn more about the interplay between O-star evolution and cluster dissolution.

Since the early-type $\mathrm{O}$ stars are more massive and have lower velocities, it is natural to consider mass segregation as the reason for these lower velocities. However, the relaxation time is of the order of $100 \mathrm{Myr}$, which is more than the maximum age of late-type $\mathrm{O}$ stars (11 Myr). So, it is unlikely that these systems have enough time to reach equipartition and mass segregation. Despite this fact, we test this idea and we find that the inferred velocities are not readily consistent with equipartition anyway. In equilibrium, the stars in a cluster are in equipartition in their kinetic energies. Therefore, the ratio of masses for two stars should equal the inverse ratio squared of their velocities: 
$m_{i} / m_{j}=\left(v_{j} / v_{i}\right)^{2}$. Comparing late to early, the ratio of masses is $m_{\text {late }} / m_{\text {early }} \sim 0.3$ and the ratio of the squared velocities is $\left(v_{\text {early }} / v_{\text {late }}\right)^{2} \sim 0.4$. This seems consistent with mass segregation. However, the other comparisons do not. For mid and early, $m_{\text {mid }} / m_{\text {early }} \sim 0.43$ and $\left(v_{\text {early }} / v_{\text {mid }}\right)^{2} \sim 0.21$, which is a factor of 2 off. The late-to-mid comparison gives $m_{\text {late }} / m_{\text {mid }} \sim 0.7$ and $\left(v_{\text {mid }} / v_{\text {late }}\right)^{2} \sim 1.85$, which is also a factor of 2 off. Furthermore, if equipartition in kinetic energy were valid, then all of these ratios should have similar values. We have yet to adequately assess the uncertainties in these ratios; that will take significant more modelling. Even so, the fairly large discrepancies seem to rule out kinetic energy equipartition in the cluster.

We can use the results in Fig. 4 to also infer that LBV isolation puts interesting constraints on their evolution. The average separation for late-type $\mathrm{O}$ stars is 0.01 deg. For LBVs, the average separation is roughly five times bigger. Dimensionally, the average separation should be proportional to the dispersion velocity and the age, $S \sim v_{d} t_{\text {age }}$. If an LBV comes from the most massive stars, then one would not expect them to have ages larger than the late-type $O$ stars. Therefore, as a conservative estimate, let us assume that an LBV is an evolved massive star that has about the same age as a late O-type star. Under this assumption, since the separations for LBVs are five times bigger than late-type O stars, this implies that the dispersal velocity is five times bigger than the late-type $\mathrm{O}$ star, which is of the order of 100 $\mathrm{km} / \mathrm{s}$. To be more quantitative, in the next sections, we extend the passive model to infer the actual dispersal velocity for LBVs. Alternatively, we consider binary scenarios that may give an explanation for the relatively large isolation for LBVs.

\section{CLUSTER DISSOLUTION WITH CLOSE BINARY INTERACTIONS}

In the previous section, we suggested that the single-star dispersal model is inconsistent with the isolation of LBVs. In this section, we put the passive single-star dispersal model to the test, and show that it is indeed inconsistent with observations. In addition, we consider models that involve binary interactions in a dispersing cluster. Our aim is to develop models to see whether binary scenarios are consistent with the LBV observed separations. At the moment, there is very little information other than the separations, so it is not worth developing an overly complex model for binary interaction. We would not be able to constrain the extra parameters of the model. Therefore, we develop the simplest binary models to constrain the data. In particular, we consider two simple models that involve binary evolution in a dispersing cluster. In the first model, we consider that an LBV is the product of a merger and is a rejuvenated star; in the second model, we consider that an LBV is a mass gainer, which would also be a rejuvenated star, and receives a kick when its primary companion explodes. See Fig. 8 and 9.

In Section 4.1, we first put together an analytic model for the average separation between two stars versus time. Then in Section 4.2 we use this model to show the inconsistency in the single-star model, and we show that LBVs are either overluminous given their mass or they are the product of a merger and are a rejuvenated star. Alternatively, in Sec-

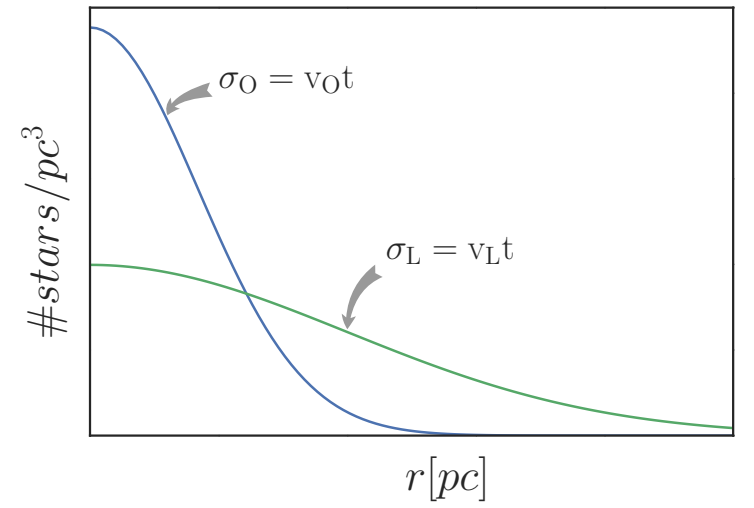

Figure 5. Two simple spatial-distribution models for the derivation of our analytic scalings.

tion 4.3, we use the analytic model to develop a kick model, and in Section 4.4, we use this model to infer a potential kick velocity for LBVs. In summary, we illustrate that the isolation of LBVs is consistent with binary scenario and is inconsistent with the single-star model.

To constrain the models, we first derive analytic scalings for the average separations, and then we explore whether these scalings are consistent with simple binary models. First, we consider simple models for the spatial distribution of two groups of stars, type $\mathrm{O}$ and type L. Each has a Gaussian spatial distribution with its own velocity dispersion $v_{d}$ , which we label as $v_{\mathrm{O}}$ and $v_{\mathrm{L}}$. Later, we will consider two scenarios: one in which these average velocity dispersions are the same, and one in which they are different. For a visual representation of these simple models, see Fig. 5. Given these distributions, we calculate the average separation between a star and the nearest star in the same group. Then we calculate the average separation between a star in group $\mathrm{O}$ and a star in group L. The average separation between stars in the same population is

$\langle S\rangle=2 \pi \int_{0}^{\infty} S p(r) r d r$,

where $S$ is the separation, and $p(r)$ is the probability density function $p(r)=\frac{1}{2 \pi \sigma^{2}} e^{-r^{2} / 2 \sigma^{2}}$ where $\sigma=v t$. To calculate the mean value of the separation, we need to find the separation $(S)$. One way to estimate the distance to the nearest neighbour is to use the spatial density of stars. In general, an estimate for the distance to the nearest neighbour is, $\hat{S} \approx 1 / n^{1 / d}$, where $n$ is the number density and $d$ is the number of dimensions that we consider (Ivezic et al. 2014). When viewing clusters projected on to the sky, $d=2$,

$\hat{S} \approx 1 / n^{1 / 2}$.

If we consider a simple density distribution, $n(r)=$ $\frac{N}{2 \pi \sigma^{2}} \exp -r^{2} / 2 \sigma^{2}$, then the average separation in twodimensional space is

$\langle\hat{S}\rangle=2\left(\frac{2 \pi}{N}\right)^{1 / 2} \sigma$,

where $N$ is the total number of stars in the cluster.

Now we consider two populations of stars. One we represent with ' $O$ ', which represents the largest number of tracer 
stars. As the label suggests, we will later consider O stars as a large number of tracer stars. The other, 'L', represents a more rare set of tracer stars, which may have a different density distribution than the first. Obviously, later ' $L$ ' will represent LBVs. In this case, the combined density is

$n_{O L}(r)=\frac{N_{O}}{2 \pi \sigma_{O}^{2}} e^{-r^{2} / 2 \sigma_{O}^{2}}+\frac{N_{L}}{2 \pi \sigma_{L}^{2}} e^{-r^{2} / 2 \sigma_{L}^{2}}$.

With this two-component expression for the density, we can evaluate the local separation, $\hat{S}$, via equation. (5) and then we can calculate the average separation from equation. (4). Calculating the average separation is numerically straightforward. However, with a small but useful assumption, we can derive an analytic estimate for the average separation. To make it easier to calculate the integral analytically, we make two assumptions. First, we assume that the average separation is roughly given by the scale of one over the square root of the average density. Therefore,

$\left\langle S_{O L}\right\rangle \approx \frac{1}{\left\langle n_{O L}\right\rangle^{1 / 2}}$.

Secondly, because LBVs are extraordinarily rare compared to the $\mathrm{O}$ stars, we assume that $N_{O} \gg N_{L}$. By considering these two assumptions, the average density is

$\left\langle n_{O L}\right\rangle \approx \frac{N_{O}}{2 \pi\left(\sigma_{O}^{2}+\sigma_{L}^{2}\right)}$.

Once we plug this into the equation for $\left\langle n_{O L}\right\rangle$, equation. (8) leads to the average distance from LBVs to the nearest $\mathrm{O}$ star:

$\left\langle S_{O L}\right\rangle \approx\left(\frac{2 \pi\left(\sigma_{O}^{2}+\sigma_{L}^{2}\right)}{N_{O}(t)}\right)^{1 / 2}$

Soon we will use the separation between O stars to help constrain the models for LBVs, so we now derive an analytic model for $\left\langle S_{O}\right\rangle$. The average density for $\mathrm{O}$ stars is

$\left\langle n_{O}\right\rangle=\frac{N_{O}(t)}{4 \pi \sigma_{O}^{2}}$,

and so the average separation between $\mathrm{O}$ stars is roughly

$\left\langle S_{O}\right\rangle \approx \frac{1}{\left\langle n_{O}\right\rangle^{1 / 2}}=\left(\frac{4 \pi}{N_{O}(t)}\right)^{1 / 2} \sigma_{O}$.

To make use of these expressions for the average separation, we need to compare the separations between two different tracer populations. Because the masses of mid-type $\mathrm{O}$ stars correspond roughly to inferred minimum mass of LBVs, we use the mid-type O-star average separation as a reference:

$\left(\frac{\left\langle S_{O L}\right\rangle}{\left\langle S_{O}\right\rangle}\right)^{2}=\frac{1}{2}\left(1+\frac{\sigma_{L}^{2}}{\sigma_{O}^{2}}\right) \frac{N_{O}\left(t_{O}\right)}{N_{O}\left(t_{L}\right)}$,

where we are careful to consider how the number of O stars changes with time and we evaluate this function at the age of the LBV population and the reference O-star population. This equation represents the general expression relating age, the average separations and the drift (or kick) velocity of each tracer population.

In the expressions for the average separations, the separations grow due to two effects: a drift velocity and the death of O stars. The drift part is simply proportional to $t$. Next, we explicitly derive the number of $\mathrm{O}$ stars as a function of time, $N_{O}(t)$. Given a mass function $d N / d M$, the total number of $\mathrm{O}$ stars is $N_{O}=\int_{M_{1}}^{M_{2}} \frac{d N}{d M} d M=\frac{A}{-\alpha+1}\left(M_{2}^{-\alpha+1}-M_{1}^{-\alpha+1}\right)$, where $M_{1}$ is the minimum mass for an $\mathrm{O} \operatorname{star}\left(\sim 16 M_{\odot}\right), M_{2}$ is the maximum mass for an $\mathrm{O}$ star, which is a function of the age of the cluster, $\alpha$ is the slope (we use Salpeter, 2.35) and $A$ is a normalization constant. If we assume a power-law relationship between mass of an $\mathrm{O}$ star and its lifetime as an $\mathrm{O}$ star, then we can relate the age of an $M_{2} \mathrm{O}$ star to the age of an $M_{1} \mathrm{O}$ star: $M_{2}=M_{1}\left(\frac{t}{t_{1}}\right)^{-1 / \beta}$. From the binary population synthesis code, BINARY_C (Izzard et al. 2004, 2006, 2009), we find that the value of $\beta \sim 1.7$. Combining these expressions, we get an equation for the number of $\mathrm{O}$ stars as a function of the age of the cluster, $t$,

$N_{O}(t)=\frac{A}{1-\alpha}\left(1-\left(\frac{t}{t_{1}}\right)^{\tau}\right) M_{1}^{1-\alpha}$.

With an explicit function for the number of O stars, we may now derive the equation relating separation, age and velocity, including explicitly all of the dependence on time. Substituting the expression for $N_{O}(t)$, equation. (14) into the general analytic expression, equation. (13), we finally arrive at the general analytic formula, explicitly relating separation, age and velocity:

$\left(\frac{\left\langle S_{O L}\right\rangle}{\left\langle S_{O}\right\rangle}\right)^{2}=\frac{1}{2}\left(1+\left(\frac{v_{L} x}{v_{O} x_{O}}\right)^{2}\right) \frac{\left(1-x_{O}^{\tau}\right)}{\left(1-x^{\tau}\right)}$,

where $\tau=\frac{\alpha-1}{\beta}, x_{O}=\frac{t_{O}}{t_{1}}$ and $x=\frac{t_{L}}{t_{1}} \cdot t_{1}(11 \mathrm{Myr})$ is the age of the minimum mass and $t_{O}(3 \mathrm{Myr})$ is a reference age. We estimate these values from binary population synthesis code, BINARY_C (Izzard et al. 2004, 2006, 2009).

In the next subsections, we use our general analytic result, equation. (15), to explore what average separations one would expect when we consider the passive dissolution in three scenarios, a single-star evolution scenario, a binary scenario that involves a merger and a binary scenario that involves a kick.

\subsection{PASSIVE MODEL}

Using our analytic estimates for the average separation, we assume that the dispersal velocities for LBVs and O stars are the same and estimate the average separation for the passive single-star model. Comparing this model to the observations, we find that the passive single-star model is inconsistent with the observations. If LBVs do passively disperse with the same velocity as the rest of the $\mathrm{O}$ stars, then we propose that LBVs are the product of a merger and are rejuvenated stars.

In this case, we need to consider the average separation when the dispersal velocities for LBVs and O stars are the same. In this scenario, our general analytic expression, equation. (15), reduces to

$\frac{S_{L}}{S_{O}}=\left[\frac{1}{2}\left(1+\left(\frac{x}{x_{O}}\right)^{2}\right) \frac{\left(1-x_{O}^{\tau}\right)}{\left(1-x^{\tau}\right)}\right]^{1 / 2}$.

This equation represents the passive model. 


\subsection{INCONSISTENCY IN THE PASSIVE MODEL IMPLIES MERGER AND REJUVENATION}

Next, we use the passively dissolving solution, equation. (16), to show that the isolation of LBVs is inconsistent with the single-star scenario. If LBVs are massive stars above $21 M_{\odot}$ and evolve as isolated stars, then Fig. 7 and Fig. 8 demonstrate that the maximum ages of these LBVs are wholly inconsistent with the large separations observed for massive stars.

The passive model predicts much lower separations than the observational data. See Fig. 6 for an illustration. The orange curve represents the passive model, $S_{L B V}$ in equation. (16). The solid brown line illustrates the LBVs' average separation obtained from the data compared to the reference average separation, $\frac{S_{L B V}}{S_{0}}$. It is clear that most of the LBVs have larger separations compared to what the passive model predicted.

Moreover, in the passive model, LBVs do not have enough time to get to the observed average separation. Fig. 7 shows the same passive model, the observed LBV separation, but this time we simplify the possible ages of LBVs by showing the ages for the average mass of our LBV sample. Clearly, if LBVs evolve as a normal single star, then they do not have enough time to reach the large separations. Instead, let us consider how old an LBV would have to be in order to passively disperse to the observed separations. Fig. 7 shows that the age would need to be about 9.2 Myr. Yet this age corresponds to the main-sequence turnoff time for a $19 M_{\odot}$ star or the death of a $21 M_{\odot}$ star. Both of these values are below the average mass of the LBVs, $50 M_{\odot}$ (Section 2). It is clear that considering LBVs in the context of a standard single-star evolution is inconsistent with the isolation.

In short, the luminosity-to-age mapping of single-star models is inconsistent with the extreme isolation of LBVs. One can consider this mapping in two steps: an age-to-mass mapping and a mass-to-luminosity mapping. Technically, the breakdown in the luminosity-to-age mapping could be a result of the breakdown in either one of these steps. In other words, LBVs could be far more luminous than their masses would suggest. At the moment, there is no known physics that would lead to this, so we instead consider how binary evolution may alter the mass-to-age mapping.

Assuming that the drift velocities of the LBVs and the $\mathrm{O}$ stars are the same, then one possible solution is that LBVs are the result of mergers and are rejuvenated stars. See Fig. 8 to visualize the merger model. We are not the first to suggest that LBVs are linked to close binary interaction. For example, see Justham et al. (2014) and Gallagher (1989). What is different here is that, following Smith \& Tombleson (2015), we analyse how the spatial distribution of LBVs strongly suggests close binary interactions.

\subsection{KICK MODEL}

Another binary model that is consistent with the isolation of LBVs is the kick model. To visualize the kick model, consider the binary scenario in Fig. 9. In this model, the primary star, the more massive star, evolves first and transfers mass to the secondary star. If the more massive star is massive enough to explode as a core-collapse SN, then the companion may receive a kick. This kick may be imparted by either an asymmetric explosion, the Blaauw mechanism (Blaauw 1961) or a combination of both. In this paper, we do not model the binary evolution and kick velocities. Rather we just assume that there are two populations, one more numerous and does not receive kicks (the $\mathrm{O}$ stars), and one that is less numerous and whose velocity distribution is dominated by kicks.

Once again, we may use our general analytic expression, relating the separations, age and velocities, equation. (15), but this time we express $v_{L}$ in terms of the age, $x=t_{L} / t_{1}$, and the measured values of the separations,

$\frac{v_{L}}{v_{O}}=\frac{x_{O}}{x}\left[2\left(\frac{\left\langle S_{O L}\right\rangle}{\left\langle S_{O}\right\rangle}\right)^{2} \frac{\left(1-x^{\tau}\right)}{\left(1-x_{O}^{\tau}\right)}-1\right]^{1 / 2}$.

Smith \& Tombleson (2015) showed that the average distance from LBVs to the nearest $\mathrm{O}$ star is $\sim 6.5$ times larger than the average distance from $\mathrm{O}$ star to the nearest $\mathrm{O}$ star. If the age of LBVs are similar to the average mid-type $O$ star, then in the assumption of the kick model, this immediately implies that $v_{\mathrm{L}}$ is roughly nine times larger than $v_{\mathrm{O}}$. In the next subsection, we estimate the LBVs' drift velocity given this model.

\subsection{ESTIMATION AND INTERPRETATION OF THE KICK VELOCITY}

Fig. 10 shows the inferred kick velocity as a function of the LBV age. If the mass gainer that eventually becomes the LBV gains little mass, then there is little discrepancy between the zero-age main-sequence mass and the final mass. In this case, there is little difference between its apparent age and its true main-sequence age. Then its true age is relatively short and the only way to get a large separation with a large kick velocity. In this scenario, we find that the kick can be as high as $105 \mathrm{~km} / \mathrm{s}$. If there is no mass gained and hence a larger kick (upper left in Fig. 10) then the star that was kicked has not necessarily had any anomalous evolution (no accretion and spin-up) and hence gives no special explanation for its observed LBV instability. On the other hand, if the mass gain is high, then the true main-sequence age would be much older than the current mass implies. With a much older age, the velocity required to get a large separation is much lower. It might even be zero, in which case, the LBV has gained so much mass that it is rejuvenated like a merger product. The horizontal black solid line represents the average observed separation for mid-type $\mathrm{O}$ stars. The solid blue line curve represents our model to infer the kick velocity, equation. (17).

Though we predict that the kick velocities may be as high as $\sim 105 \mathrm{~km} / \mathrm{s}$, we note that the kick may be quite low, even near zero. Humphreys et al. (2016) argued that none of the LBVs in the LMC have high velocities. They suggest that most of the LBV velocities [listed in table 3 of Humphreys et al. (2016)] are consistent with the systemic velocities of the LMC, concluding that the observed velocities are inconsistent with the kick. In Fig. 10, we show that the kick velocity may be anywhere from 0 to $\sim 105 \mathrm{~km} / \mathrm{s}$ depending on the orbital parameters at the time of the SN, and how much mass was transferred. To further constrain the mass-gainer and kick model, one will need to properly model binary evolution including explosions and kicks in the 


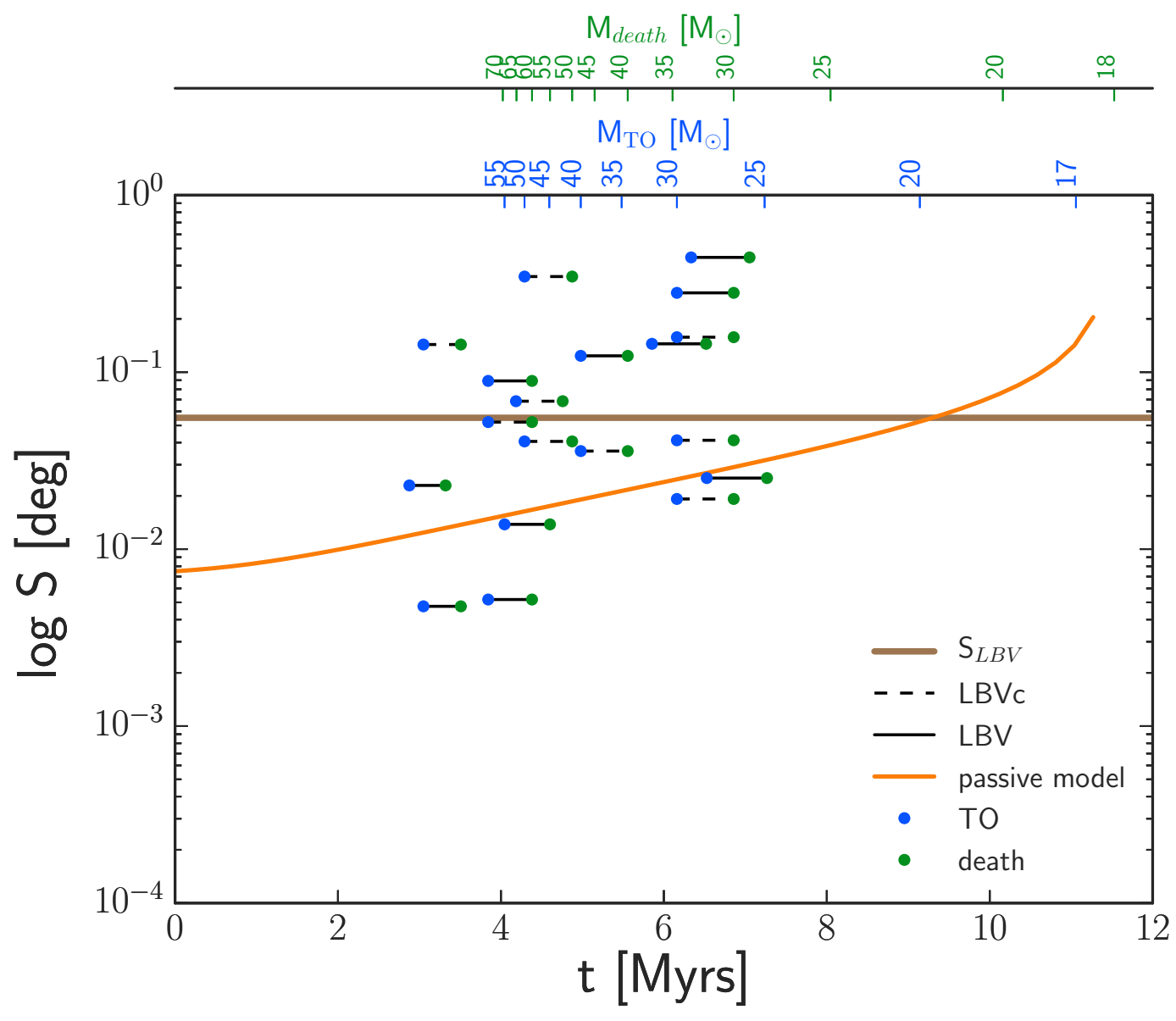

Figure 6. LBV isolation is inconsistent with the single-star model and passive dissolution of the cluster. The solid brown line shows the LBVs' average separation obtained from the data. The orange curve shows our analytic description, equation. (16), for the average separation in the context of passive dissolution. This model requires a reference; we used the mid-type O observations as the reference. The line segments show the purported masses and allowable ages for the LBVs (solid segments) and LBV candidates (dashed segments). If LBV mass estimates are correct, then even when one considers the maximum age for LBVs, the separations are much larger than what the single-star passive model predicts.

context of dispersing cluster. Current modelling efforts already indicate that the dispersal velocities from binary evolution could have a large range, even low dispersal velocities (Eldridge et al. 2011; de Mink et al. 2014; Smith 2016). However, putting these binary models in the context of cluster dispersion is yet to be done. For now, we present the scale of the problem; in a subsequent paper, we will model the distribution of observed velocities one would expect.

\section{SUMMARY}

Smith \& Tombleson (2015) found that LBVs are surprisingly isolated from other $\mathrm{O}$ stars. They suggested that the relative isolation is inconsistent with a single-star scenario in which the most massive stars undergo an LBV phase on their way to evolving into a WR star. Instead, they suggested that a binary scenario is likely more consistent with the relative isolation of LBVs. In this paper, we test this hypothesis by developing crude models for single-star and binary scenarios in the context of cluster dissolution. Even with these crude models, we find that the LBVs' isolation is mostly inconsistent with the standard passive single-star evolution model. In particular, if LBVs do evolve as single stars, then their isolation implies an age that is twice the maximum age of an average LBV. It may be the case that a small fraction of LBVs could evolve as single stars and still be consistent with the measured isolation. However, the fact that most LBVs are very isolated suggests that a large fraction is inconsistent with single-star evolution. For most LBVs, there is a clear problem in the single-star model's mapping between luminosity and kinematic age, and this is either because there is a problem in the luminosity-to-mass mapping or there is a problem in the mass-to-age mapping. In this paper, we consider how binary evolution might affect the latter, the mass-to-age mapping. We find that the LBV isolation is most consistent with two binary scenarios: either LBVs are mass gainers and receive a kick anywhere from 0 to $\sim 105 \mathrm{~km} / \mathrm{s}$ or they are the product of mergers and are rejuvenated stars. Of course, LBVs may actually represent a combination of these two scenarios. Based on their envi- 


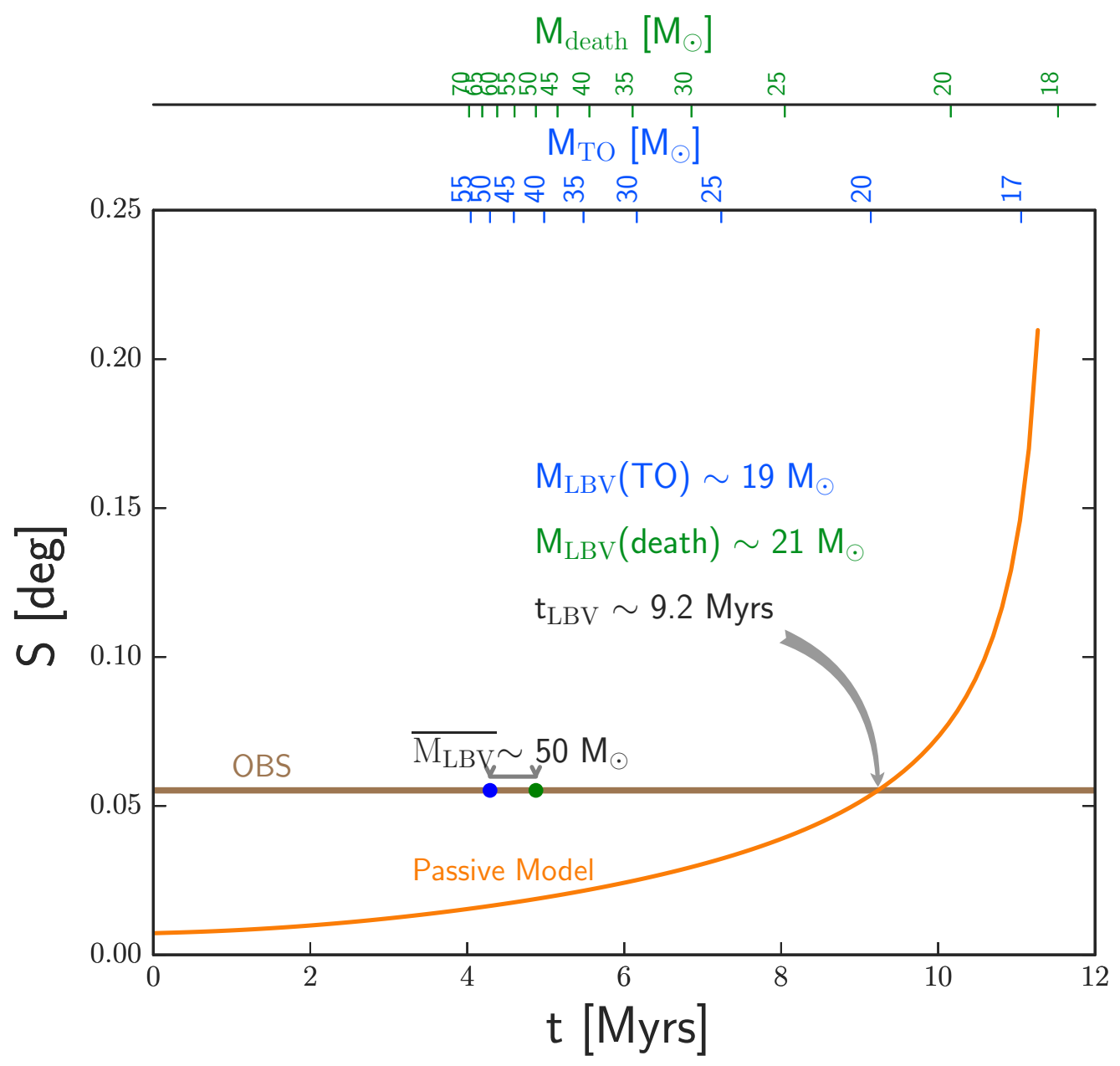

Figure 7. The relative isolation of LBVs is consistent with a binary merger in which the LBV is a rejuvenated star. The solid brown line shows the observed LBV average separation. If LBVs passively dissolve with the rest of the cluster (orange curve), then we infer an average age for LBVs of $9.2 \mathrm{Myr}$. This corresponds to the main-sequence turn-off time for a $19 M_{\odot}$ star and the death time for a 21 $M_{\odot}$ star. However, the average mass for LBVs estimated from their luminosities is roughly $50 M_{\odot}$. Stars this massive do not live long enough to passively disperse to large distances. On the other hand, if LBVs are the products of a merger, and the primary has a mass between about 19 and $21 M_{\odot}$, then the rejuvenated star could have a high luminosity, high mass and old age allowing it to disperse to larger distances.

ronments, it is quite possible that some are mass gainers and some are the product of mergers.

In order to constrain these models, we first reproduce the results of Smith \& Tombleson (2015). Similarly, we obtain the position of all $\mathrm{O}$ stars within $10^{\circ}$ projected radius of 30 Dor, and we construct distributions of distances to the nearest $\mathrm{O}$ star. Our distributions are very similar to theirs. We find that the distributions for the LBVs and $\mathrm{O}$ stars are very unlikely to be drawn from the same parent distributions. In particular, the average distance to the nearest $\mathrm{O}$ star is $\sim 6.5$ times larger for LBVs than $\mathrm{O}$ stars. To better inform our models, we further characterize the distributions and find that all of the nearest neighbour distributions are lognormal. The fact that the distributions are simple and lognormal demands that our models for cluster dissolution are also simple.

We propose simple Monte Carlo and analytic models for the dispersal of open clusters of $\mathrm{O}$ stars. In this model, we sample from distributions of cluster sizes, the Salpeter IMF for stars and random ages. To match the observed separations for early $\mathrm{O}$ stars, we find that the early-type clusters need a drift velocity of the order of $7 \mathrm{~km} / \mathrm{s}$. For the mid and late types, we require drift velocities of the order of 15 and 11 $\mathrm{km} / \mathrm{s}$, respectively. The higher drift velocities for later type $\mathrm{O}$ stars hint that binarity and kicks may play a prominent role in cluster dissolution. In fact, some fraction of later type $\mathrm{O}$ stars may be mass gainers or the product of mergers. In a future paper, we will investigate whether one can constrain the fraction of kicks and strong binary interaction.

Using the results of the Monte Carlo simulations as a guide, we develop an analytical model for the average separation as a function of drift velocity and time. These analytic scalings strongly suggest that LBV isolation is inconsistent with single-star stellar evolution. Instead, these scalings in combination with LBV isolation suggest that either LBVs have lower initial masses (and hence longer lifetimes) than one would infer from luminosities or the isolation is most consistent with some sort of binary interaction: either 


\section{Merger Model}
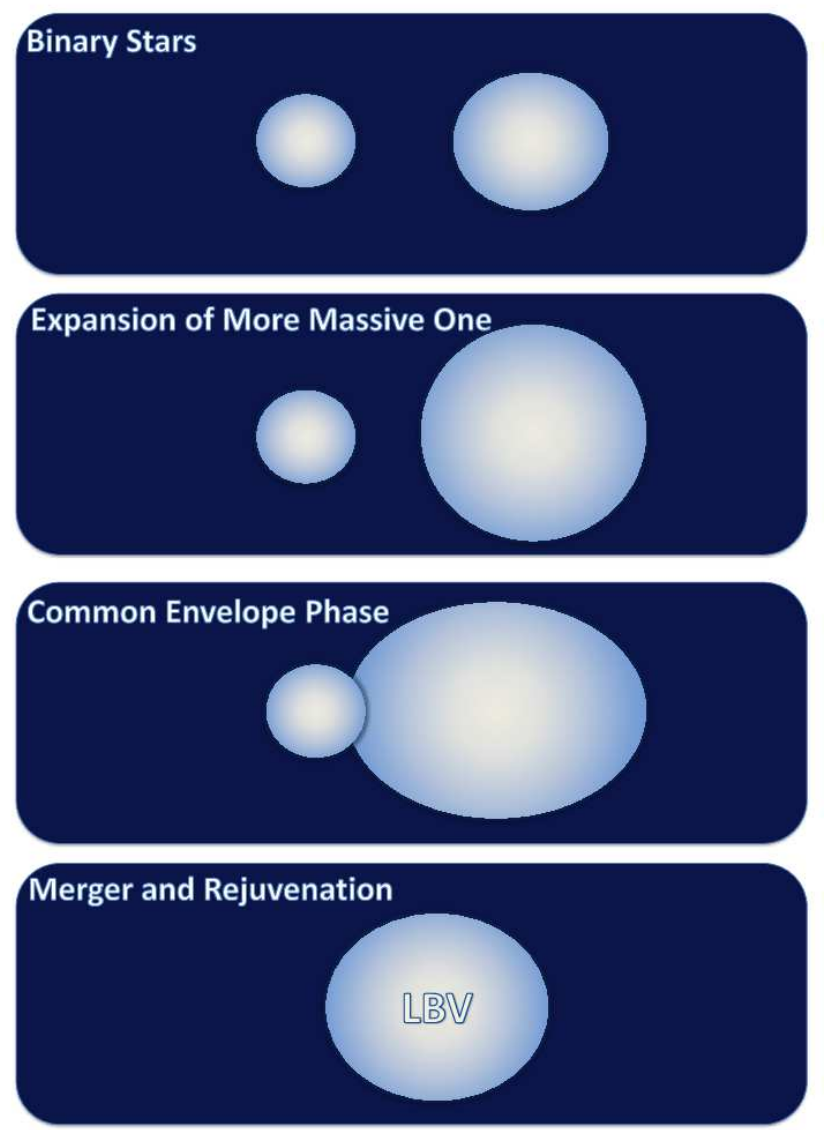

Figure 8. Merger model outline. In this binary scenario, LBVs are a product of rejuvenation of two massive stars. For a given mass, a rejuvenated star has a larger maximum possible age than a single-star counterpart. These larger maximum ages allow a rejuvenated star enough time to drift farther from other $\mathrm{O}$ stars. This is one binary scenario that is consistent with the isolation of LBVs.

a merger or kick. If LBVs have the same dispersion velocity that we infer from mid-type $\mathrm{O}$ stars, then the time to get to the relatively large isolation is $9.2 \mathrm{Myr}$. However, the average mass of LBVs is $50 M_{\odot}$ which has a maximum time of $\sim 4.8$ Myr. This is clearly inconsistent. On the other hand, binary interactions can easily achieve large isolations. In one scenario, LBVs might be the product of the merger of two massive O-type stars, in which the primary has a mass of at least about $19 M_{\odot}$. Another possibility is a kick due to binary evolution. In this binary scenario, the less massive stars (pre-LBV stars) gain mass from its companion. After mass transfer, the primary explodes as an $\mathrm{SN}$ and the LBV receives a kick anywhere from 0 to $\sim 105 \mathrm{~km} / \mathrm{s}$.

With current observations and theory, either binary model is consistent with the data. To further constrain which binary model is most consistent, we need to gather more data and develop better models. For example, detailed kinematic observations and theory would help to distinguish between these two models. Humphreys et al. (2016) suggest that the velocities of LBVs are too low to be consistent with

\section{Kick Model}
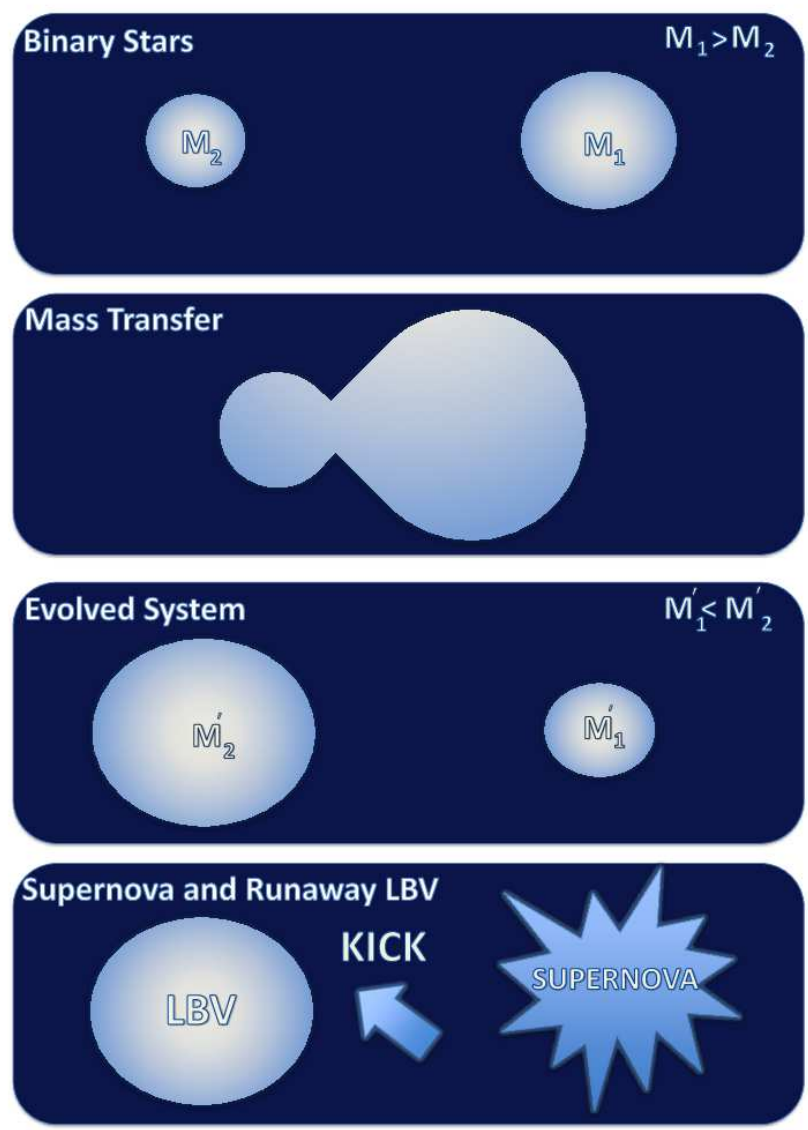

Figure 9. Kick model outline. In this binary scenario, a preLBV star gains mass from its more massive companion star. After mass transfer, the mass gainer (LBV) receives a kick when its companion explodes in an SN.

the kick scenario. However, there are binary scenarios that would produce low kick velocities. For example, if the secondary accretes so much mass that it becomes the more massive star in the binary, then this much more massive secondary will have a low orbital velocity in its binary orbit. When the low-mass primary explodes, the mass gainer drifts away at its low orbital speed. Hence, Smith (2016) pointed out that large kick speeds are not necessarily expected, especially when there has been a significant amount of mass gained (Eldridge et al. 2011; de Mink et al. 2014). We show that the mass-gainer scenario currently predicts a wide range of kick velocities. To truly test the consistency of the kick model, we must first model binary evolution and develop a model for the appearance of the kinematics, including randomness, projection, etc. The merger model would manifest as an inconsistency between the maximum age of the LBV and the surrounding stellar population. Therefore, to constrain the merger model, we need better mass estimates for the LBVs and age estimates for the surrounding stellar populations.

In conclusion, we develop models for cluster dissolution and the spatial distribution of LBVs and $\mathrm{O}$ stars. These models suggest that single-star evolution in passively evolv- 


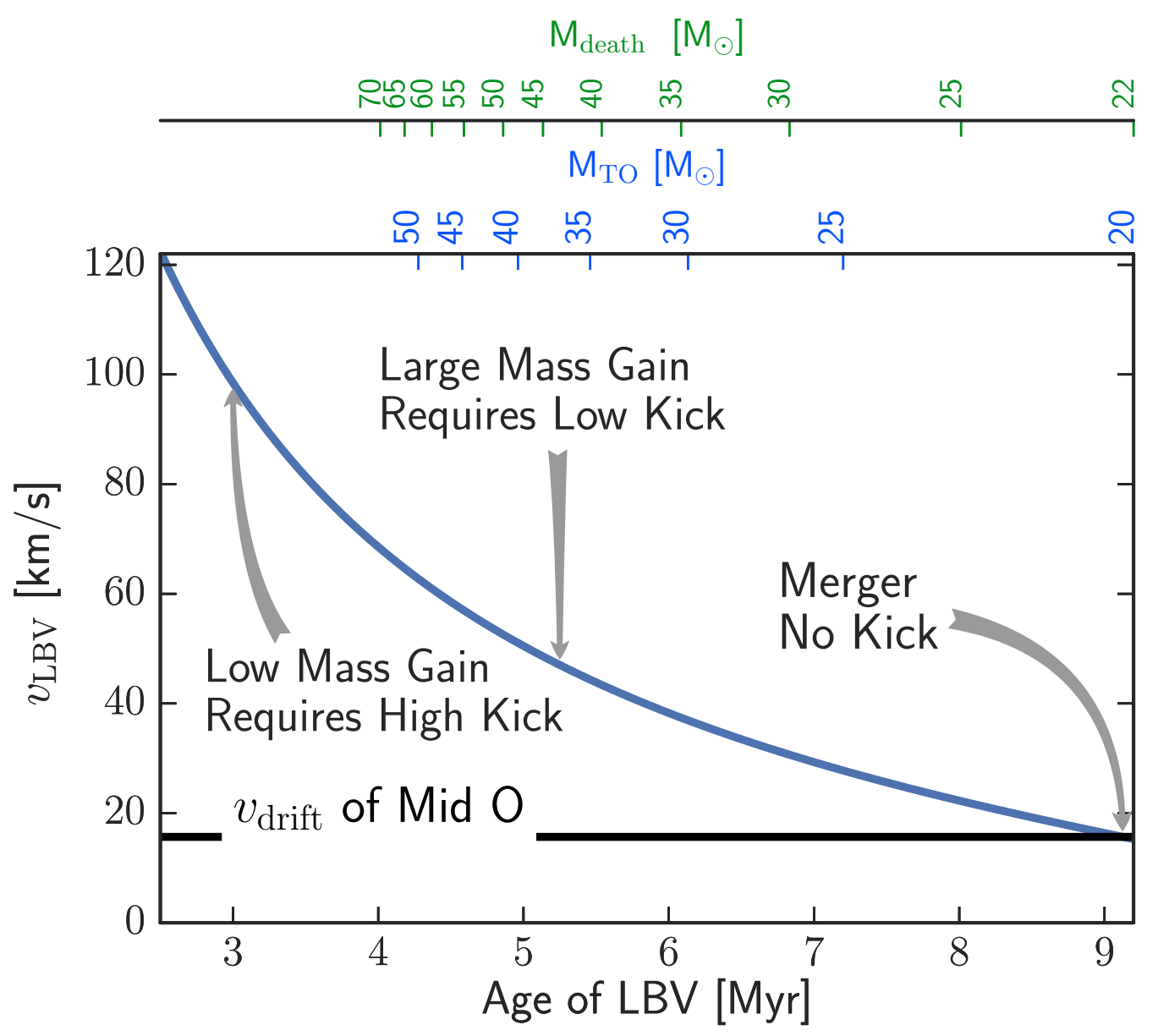

Figure 10. LBV dispersion velocity as a function of LBV age. Another binary model that is consistent with observations is one in which the LBV is a mass gainer and receives a kick when its primary companion explodes. The solid blue curve represents our analytic model, equation. (17). For reference, the solid black line shows the drift velocity of mid-type O stars, see Fig. 4. As a mass gainer, the age of the LBV will be older than one would infer from its luminosity and mass. If LBVs gain little to no mass, then the kick required to match the observed separations is in the range $0-105 \mathrm{~km} / \mathrm{s}$. The lower end of this range corresponds to high mass transfer. Specifically, if LBVs have an age of the order of 9.2 Myr, then we suggest that LBVs are mergers and received no kick.

ing clusters is inconsistent with the extreme isolation of LBVs. Instead, we find that either LBVs are less massive than their luminosities would imply or binary interaction is most consistent with LBV isolation. In particular, we crudely find that two binary scenarios are consistent with the data. Either LBVs are mass gainers and received a kick when the primary exploded or they are rejuvenated stars, being the product of mergers.

\section{ACKNOWLEDGMENTS}

This research has made use of the SIMBAD data base, operated at CDS, Strasbourg, France. Support for NS was provided by the National Science Foundation (NSF) through grants AST-1312221 and AST-1515559 to the University of Arizona.

\section{REFERENCES}

Blaauw A., 1961, Bull. Astron. Inst. Neth, 15, 265 Bohannan B., Walborn N. R., 1989, PASP, 101, 520

Eldridge J. J., Langer N., Tout C. A., 2011, MNRAS, 414, 3501

Elmegreen B. G., Efremov Y. N., 1997, ApJ, 480, 235

Gallagher J. S., 1989, in Davidson K., Moffat A. F. J., Lamers H. J. G. L. M., eds, Astrophysics and Space Science Library Vol. 157, IAU Colloq. 113: Physics of Luminous Blue Variables. pp 185-192, doi:10.1007/978-94-009-1031-7_22

Gräfener G., Owocki S. P., Vink J. S., 2012, A\&A, 538, A40

Groh J. H., Hillier D. J., Damineli A., Whitelock P. A., Marang F., Rossi C., 2009, ApJ, 698, 1698

Humphreys R. M., Davidson K., 1994, PASP, 106, 1025

Humphreys R. M., Weis K., Davidson K., Gordon M. S., 2016, preprint, (arXiv:1603.01278)

Ivezic Z., J. Connolly A. ., T VanderPlas J. ., Gra A., 2014, Statistics, Data Mining, and Machine Learning in Astronomy. Princeton Series in Modern Observational Astronomy, Princeton University Press

Izzard R. G., Tout C. A., Karakas A. I., Pols O. R., 2004, MNRAS, 
350,407

Izzard R. G., Dray L. M., Karakas A. I., Lugaro M., Tout C. A., 2006, A\&A, 460, 565

Izzard R. G., Glebbeek E., Stancliffe R. J., Pols O. R., 2009, A\&A, 508, 1359

Justham S., Podsiadlowski P., Vink J. S., 2014, ApJ, 796, 121

Klein C. R., Cenko S. B., Miller A. A., Norman D. J., Bloom J. S., 2014, preprint, (arXiv:1405.1035)

Kotak R., Vink J. S., 2006, A\&A, 460, L5

Krumholz M. R., Tan J. C., 2007, ApJ, 654, 304

Kudritzki R.-P., Puls J., 2000, ARA\&A, 38, 613

Maíz Apellániz J., et al., 2013, in Massive Stars: From alpha to Omega. p. 198, http://a2omega-conference.net

Marconi M., Clementini G., 2005, AJ, 129, 2257

Martins F., Palacios A., 2013, A\&A, 560, A16

Martins F., Schaerer D., Hillier D. J., 2005, A\&A, 436, 1049

Meynet G., Maeder A., 2005, A\&A, 429, 581

Ofek E. O., et al., 2013, Nature, 494, 65

Owocki S. P., Gayley K. G., Shaviv N. J., 2004, ApJ, 616, 525

Puls J., Vink J. S., Najarro F., 2008, A\&AR, 16, 209

Smith N., 2014, ARA\&A, 52, 487

Smith N., 2016, MNRAS, 461, 3353

Smith N., Owocki S. P., 2006, ApJ, 645, L45

Smith N., Stassun K. G., 2017, AJ, 153, 125

Smith N., Tombleson R., 2015, MNRAS, 447, 598

Smith N., et al., 2007, ApJ, 666, 1116

Smith N., et al., 2008, ApJ, 686, 485

Smith N., Li W., Silverman J. M., Ganeshalingam M., Filippenko A. V., 2011, MNRAS, 415, 773

Vink J. S., 2012, in Davidson K., Humphreys R. M., eds, Astrophysics and Space Science Library Vol. 384, Eta Carinae and the Supernova Impostors. p. 221 (arXiv:0905.3338), doi:10.1007/978-1-4614-2275-4_10

Vink J. S., de Koter A., Lamers H. J. G. L. M., 2001, A\&A, 369,574

Walborn N. R., 1977, ApJ, 215, 53

Walker A. R., 2012, Ap\&SS, 341, 43

Weis K., 2003, A\&A, 408, 205

Wolf B., 1989, A\&A, 217, 87

Woosley S. E., Heger A., 2015, in Vink J. S., ed., Astrophysics and Space Science Library Vol. 412, Very Massive Stars in the Local Universe. p. 199 (arXiv:1406.5657), doi:10.1007/978-3-319-09596-7_7

Woosley S. E., Heger A., Weaver T. A., 2002, Reviews of Modern Physics, 74, 1015

de Koter A., Lamers H. J. G. L. M., Schmutz W., 1996, A\&A, 306,501

de Mink S. E., Sana H., Langer N., Izzard R. G., Schneider F. R. N., 2014, ApJ, 782, 7

van Genderen A. M., 2001, A\&A, 366, 508 